\title{
The Influence of Solar Activity and Cosmic Rays on Terrestrial Clouds and Global Warming
}

\author{
E. Pallé Bagó \& C. J. Butler \\ Armagh Observatory, College Hill, BT61 9DG. Armagh, N. Ireland
}

\begin{abstract}
We analyse the new ISCCP (International Satellite Cloud Climatology Project) D2 cloud data to ascertain whether or not a connection between cosmic ray flux and cloud cover exists. Our results indicate that only the low-level cloud follows solar activity over the full period, 1983-1994. Using several proxies for solar activity and the radiative forcing for the ISCCP cloud types, we estimate the possible impact that such a solar-terrestrial connection may have on climate. We conclude that, possibly excluding the most recent decades, much of the warming of the past century can be quantitatively accounted for by the direct and indirect effects of solar activity.
\end{abstract}

\section{Introduction}

The extent to which the recent global warming has an anthropogenic origin (e.g. via the enhanced greenhouse effect) as opposed to a natural origin (e.g. through volcanic activity or solar variability) is of crucial importance for our understanding of how the Earth's climate has varied in the past and how it may vary in future. Detailed fits of global and hemispherical temperatures since the mid-19th century with empirical models involving the enhanced greenhouse effect and solar variability require at least one parameter linked to solar activity for a satisfactory fit in the mid-20th century (Kelly and Wigley, 1992; Soon et al. 1996).

A modulation of the Earth's albedo by changes in cloudiness resulting from changes in the flux of galactic cosmic rays, as a mechanism for solar-climate relationship was proposed by Svensmark and Friis-Christensen (1997). This last mechanism looked particularly promising after a strong correlation between cloud factor over mid-latitude oceans and cosmic ray flux was found by these authors for the period 1984-1991.

\section{Correlation between cosmic ray flux and cloud factors}

Our first objective was to ascertain whether or not previous reports (Svensmark and Friis-Christensen, 1997; Svensmark, 1998), that the total cloud cover over mid-latitude oceans, excluding the tropics, (hereafter SFC zones) correlates strongly with cosmic ray flux, are substantiated by the new ISCCP D2 data. In Fig. 1 we plot the total cloud cover over this latitude range together with the cosmic ray flux, suitably scaled for overlap. 


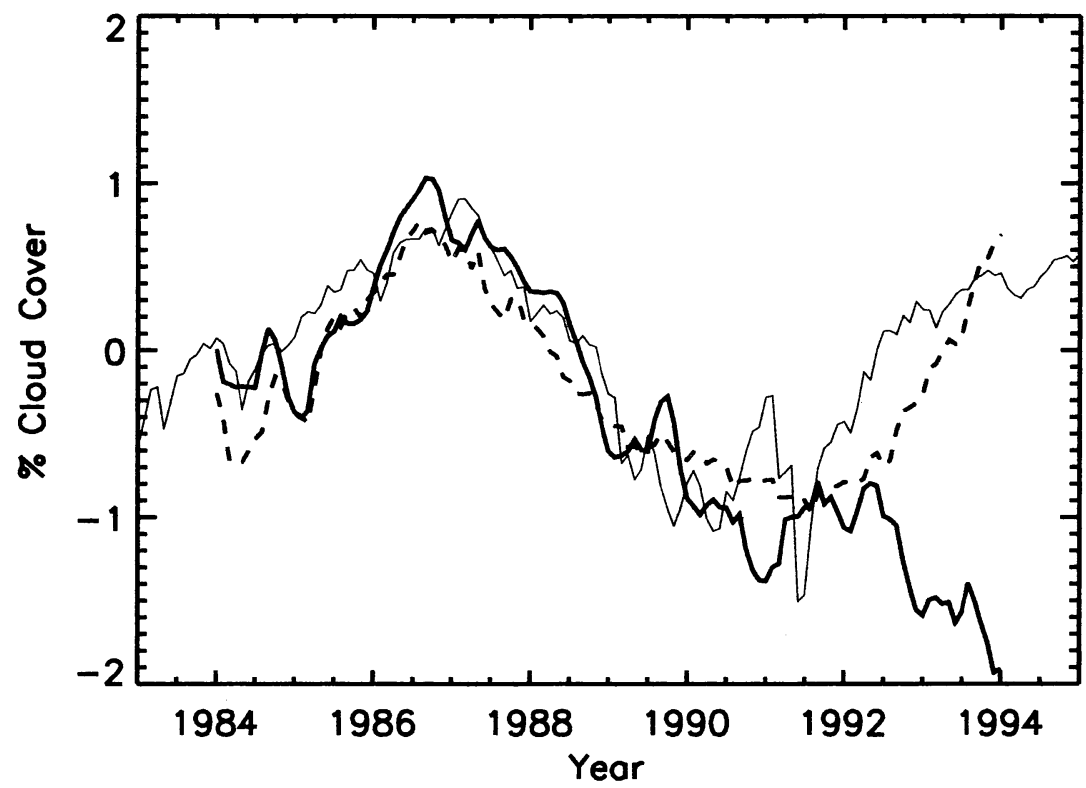

Figure 1. Total cloud cover (solid line) and low cloud cover (broken line) over the SFC areas as obtained with the D2 dataset from infrared (low clouds) and visual plus infrared (total cloud) observations. The thin line represents the Climax Cosmic Ray Flux (CRF) scaled for comparison.

In Fig. 2, we show the mean monthly cloud factors for selected regions for low, medium and high altitude clouds $(2,4.5$ and $10 \mathrm{~km}$ mean altitude respectively), each smoothed with a twelve-month filter to eliminate seasonal effects. We note the following: (1) the good correlation between total cloud factor and cosmic ray flux from 1983-91 breaks down after 1991; (2) the high level and mid-level clouds show no systematic variation over the period 198394; (3) the low-level clouds for all latitude zones excluding the poles are well correlated with the cosmic ray flux over the period 1983-94.

\section{Estimating cloud forcing}

The role of clouds in climate is still not well understood; they have two opposite effects. On the one hand they tend to cool the climate by reflecting short-wave solar radiation back to space, and on the other to warm the climate by trapping the long-wave radiation emitted from the Earth's surface. The balance of these two effects is in part determined by the cloud height; on average low clouds are believed to cool and high clouds to warm the climate (Ockert-Bell \& Hartmann, 1992; Ramanathan et al., 1989). Provided the above correlation is maintained over long periods, a reduced low-cloud factor would be expected during high solar activity, and the increased solar activity in recent decades would translate 
into a global decrease in the low, cooling clouds thereby contributing to global warming (Svensmark, 1998).
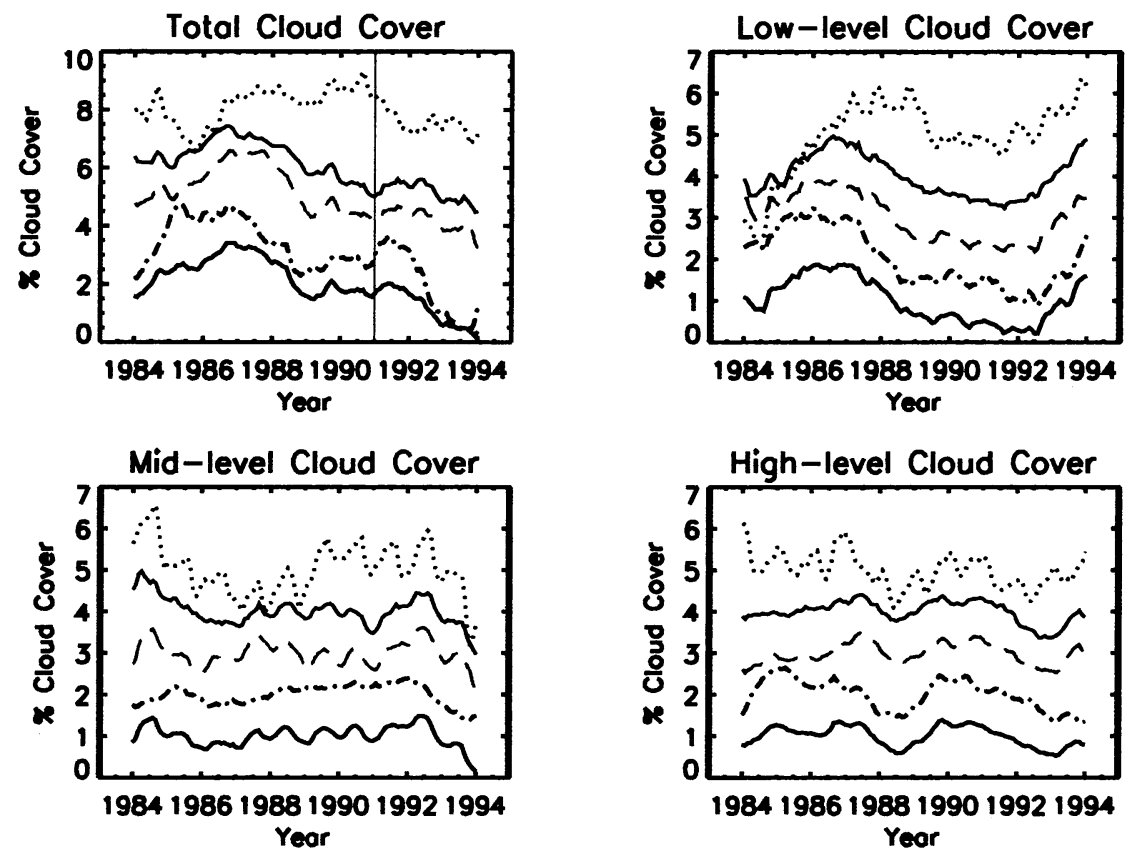

Figure 2. The 12-month running mean of the total, low-level, mid-level and high-level cloud cover for the period 1983-1995 covered by the ISCCP D2 dataset. Five different series are represented in each panel, bottom to top as follows: Cloud cover over the whole Earth (low solid line), the tropics (dashed-dotted line, $\pm 22^{\circ} .5$ ), mid-latitudes (dashed line, $\pm 22^{\circ} .5 \Rightarrow \pm 60^{\circ}$ ), the SFC zones (upper solid line, ocean areas excluding the tropics) and the poles (dotted line, $\pm 60^{\circ} \Rightarrow \pm 90^{\circ}$ ). Cloud cover is measured as the fraction of the sky covered by clouds. The amplitude of the cloud cover variation is real, but the mean value is shifted for plotting purposes.

In order to make a prediction of the low cloud factor, earlier in the 20th century and in the 19th century when cosmic ray fluxes are unavailable, we have determined the regression relations between the Sunspot Number, the <aa> index and the low cloud factor as determined from the ISCCP-D2 data. For even longer periods, extending back before the 19th century, we use the Heliocentric Potential, an interplanetary magnetic field index calculated by O'Brien (1979) which is based on the carbon isotope concentration in tree-rings.

From the regression between the various activity indices and the low cloud factor over the interval 1983-1994, we can make a prediction of the change in average low cloud factor since the late 19th century. The results indicate a decrease in low cloud factor by about $1 \%$ over the past one hundred years, leading to a reduced albedo and positive radiation forcing in recent decades. Together with the increased forcing from the increase in solar irradiance, this leads to a total solar activity-induced change in the global mean temperature of 
$\sim 0.5^{\circ} \mathrm{C}$ which is close to the observed increase of $0.55^{\circ} \mathrm{C}$ since 1900 (Lean \& Rind, 1998; Jones \& Briffa, 1992).

Similarly, we can compute from the change in ${ }^{14} \mathrm{C}$ levels, (via the Heliocentric Potential), the effect of activity-induced cloudiness on temperatures in the late 17 th and early 18th centuries during the Maunder Minimum. We derive a cloud induced global cooling of $0.5^{\circ}$ during this period compared to modern temperatures. This together with a cooling of $0.32^{\circ} \mathrm{C}$ from an inferred reduced solar irradiance of $\sim 0.25 \%$ at that time (Rind, 1993) implies a combined cooling of $0.82^{\circ} \mathrm{C}$, reasonably close to the value of $\sim 1.0^{\circ} \mathrm{C}$ believed to have occurred at this time.

The details of these calculations are given in Palle \& Butler (2000). They are subject to several assumptions, including: (1) the correlation between low cloud and cosmic ray flux is maintained over long time scales; (2) other cloud types remain constant; (3) that there is no additional change in the cloud factor from global warming, i.e. no feedback.

Note: The full text of this paper will appear in the August 2000 issue of Astronomy \& Geophysics, Journal of the RAS. Vol 41, Issue 4, 18-22 (Pallé and Butler, 2000).

Acknowledgments. Research at Armagh Observatory is grant-aided by the department of Culture, Arts and Leisure for N. Ireland.

\section{References}

Jones, P.D., Briffa, K.R., 1992. The Holocene 2, 165-179.

Kelly, P.M., Wigley, T.M.L., 1992. Nature 360, 328-330.

Lean, J., Rind, D., 1998. Journal of Climate 11, 3069-3094.

O'Brien, K., 1979. J. Geophys. Res. 84, 423.

Ockert-Bell, M.E., Hartmann, D.L., 1992. Journal of Climate 5, 1157-1171.

Pallé, E., Butler, C.J., 2000. Astronomy \& Geophysics, 41, 18-22.

Ramanathan, V.,et al., 1989. Science 243, 52.

Rind, D., Overpeck, J., 1993. Quat. Sci. Rev. 12, 357-374.

Soon, W.H., Posmentier, E.S., Baliunas, S.L., 1996. Astrophys. J. 472, 891-902.

Svensmark, H., Friis-Christensen, E., 1997. J.A.S.T.P. 59, 1225-1232.

Svensmark, H., 1998. Phys. Rev.Lett. 81, 5027-5030. 